\title{
Big Data in Chinese Government Governance: Analysis of Decision-Making Model Innovation and Practice
}

\author{
Peng Wang, Bin Lu \\ Beijing Academy of Social Sciences Management Institute, Renmin University of China Hillhouse Academy, Beijing, China \\ Email: wangpeng@egovernment.gov.cn
}

How to cite this paper: Wang, P. and Lu, B. (2018) Big Data in Chinese Government Governance: Analysis of Decision-Making Model Innovation and Practice. Journal of Computer and Communications, 6, 129-142. https://doi.org/10.4236/jcc.2018.612013

Received: October 11, 2018 Accepted: December 23, 2018 Published: December 26, 2018

\begin{abstract}
The 19th National Congress of the Communist Party of China has put forward higher requirements for Chinese government governance. The government governance has developed to a higher stage. Meanwhile, it faces more challenges, like lack of top-level design and information sharing. To develop a government governance decision-making innovation model, we should make good use of big data to mine in the grassroots government data management network. Both the characteristics of the times and the experience of the practice have proven that big data can empower government governance and promote the construction of a service-oriented government.
\end{abstract}

\section{Keywords}

Big Data, Government Governance, Model Innovation

\section{The Situation and Problems the Chinese Government Governance Are Facing}

With the rapid development of China's new industrialization, Informatization, urbanization, and agricultural modernization, China has profound changes profound changes in economic structure, adjustment of interest patterns, ideological concepts, and social structure [1]. Chinese government governance now faces a series of new challenges and requirements. However, there is still a way to go before the Chinese government's governance' target is achieved. Chinese government governance still has problems in the government decision-making process, which are reflected in the following three aspects:

First, limitation of rational decision-making and precise decision-making. First of all, the current decision-making model for most government local affairs 
is still the elite decision-making model. The phenomenon of one-size-fits-all and deaf to the masses and expert opinions still exists. Secondly, in some areas, the lack of information and the low utilization rate of information, the incomplete effective reference information in decision-making happens. And the phenomenon of one-leaf and biased decision-making has occurred from time to time. At the same time, the government lacks political responsiveness and a comprehensive grasp of public opinion.

Second, lack of top-level design and local governments coordination. The most intuitive manifestation of the problem is that the intelligent platforms of local governments are usually not compatible. There is no unified standard for data format, quality standards, data readability, and interoperability.

Third, the feedback information is incomplete and fails to form a closed loop of effective decision-making. The times are the people who roll out, the government is the answerer, and the people are the reviewers.

Therefore, it can be seen that the current problems of government governance mainly exist in two levels, information processing and sharing awareness. While Big data can coordinate the whole decision-making process in the process of government governance with its characteristics of sharing, unity, comprehensiveness, and intelligence. Some China's local governments have also taken measures to promote a series of government innovation practices based on big data, such as smart cities and "Internet + government affairs". The characteristics of the times and the exploration of practice show that Big Data will play a vital role in the future Chinese government governance.

\section{The Innovation of Government Governance Decision-Making Model Based on Big Data}

The combination of big data and government governance is to integrate big data into every step of decision-making. Using big data to make up for the deficiencies of manpower, intelligence and link connections, to form a complete, unified and standardized decision-making model. Public derivative big data is the embodiment of big data in the field of government governance. Dealing with the use of public derivative big data in government governance decision-making procedure can empower the government governance decision-making innovation model.

Starting from the need to solve problems in government governance, the innovation of government governance decision-making model based on public derivative big data is mainly divided into the following three links [2]:

First, using the big data analysis method to detect public opinion hotspots. That is, put the multiple original and different structural network public opinion, policy texts and system-generated data, which the government faces when dealing with public affairs, as main objects. To explore text mining methods that have better analysis and interpretation effects, and combine these methods with other multi-methods and tools. To make policy hotspots consistent with find public opinion and people concerns hotspots. And selecting decision-making 
topics from the perspective of the people, to build a service-oriented government.

Second, discovering knowledge about specific policy scenarios to assist decision making. That is, through the comprehensive analysis of multi-source data, to find out the knowledge that is vital to public managers around specific public management and public policy issues. Therefore, it can help to reveal the feasible path methods driven by big data analysis to drive government decision-making optimization.

Third, using Big data simulation to drive performance evaluation and re-decision. Through the basic methods of social computing based on multi-agent simulation and so on, try to conduct a medium- and long-term evaluation of the common selection of common public issues and the decision-making effect. Based on this, the process of data analysis-driven decision-making is modularized and instrumentalized, and the government decision-making process is more scientific. Therefore, the sample survey method based on past policy evaluation is transformed into a comprehensive analysis method based on big data. A more realistic and representative policy evaluation result is obtained, which provides a scientific basis for policy revision.

Thus, in this cyclic process big data promotes a decision-making closed loop and form a complete decision-execution-evaluation-re-decision procedure. It can significantly improve the accuracy, effectiveness, timeliness and preventiveness of government governance with the awareness of big data sharing and governance. In order to maximize the effectiveness of this decision-making model, the top-level design should be done. That is, based on big data sharing, establishing a public governance platform, integrating data from various departments and regions, and developing from data integration and data fusion to data joint service, to provide the most comprehensive and broadest data support for government governance model (Figure 1).

\section{Analysis of the Innovative Application of Big Data in Government Governance}

For now, there are two directions in the innovative application of big data in

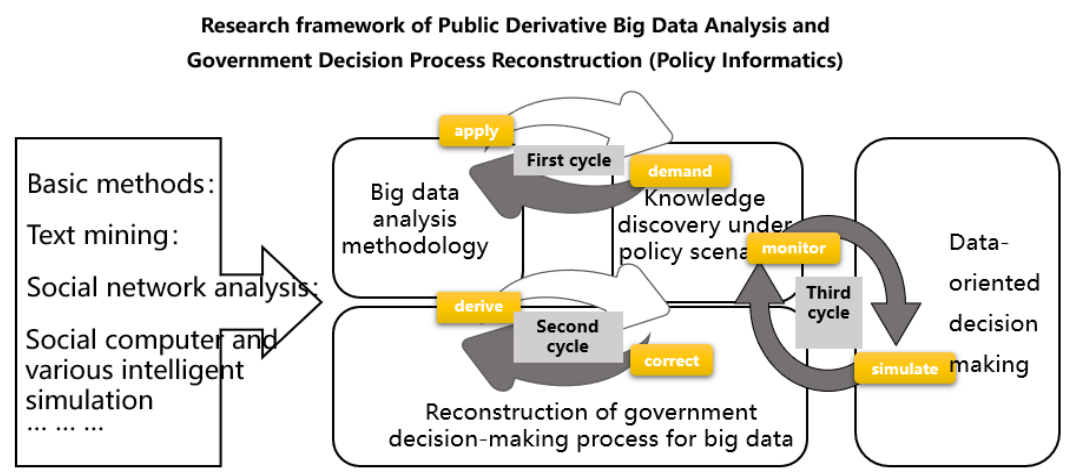

Figure 1. Government governance scientific decision support system-integration-intelligence-interaction decision support system. 
government governance decision-making model: one is the discovery and feedback of public opinions and people's livelihood and the other is the linkage management and application of the big data in grass-roots government [3].

\subsection{Research about Interaction between Government and Citizens}

Government responsiveness is the extent to which government actions are consistent with citizen opinions (Roberts and Kim, 2011: 819-839). It reflects the influence of citizens. It provides an important perspective for understanding the interaction between citizens and government. The application of big data in the study of political and civil interactions is actually the implementation of the selection process of the big data governance decision-making model innovation. Therefore, we can evaluate government responsiveness and promote big data democracy through the big data analysis methods in analyzing public opinion hotspots and detecting public opinion.

This paper selects the data from the "local leadership message board" of the online platform for political participation in People's Daily Online, to analyze the hotspots and explore the interaction between the government and citizens. The platform was commissioned in 2006 and officially launched in 2008. It is the only national network policy platform with extensive attention and exposure. It is not controlled by local governments, which greatly reduces the selectivity of local government netizens opinions and presents an objective and comprehensive political interaction process of the online political platform in the country.

This paper uses the big data analysis method to carry out text analysis, sentiment analysis and spatiotemporal analysis. Table 1 [4] presents the amount and proportions of public claims on 13 public issues from 2006 to 2014. Among them, the three topics of rural agriculture, employment and corruption attract the most public attention, with more than 60,000 posts, accounting for $32.5 \%$, $32.1 \%$ and $29.5 \%$ of the total postings respectively. They are followed by corporate affairs, municipal construction, transportation, and education, with a number of 40,000 to 50,000 postings. The three issues of environmental protection, land acquisition and social security were also paid attention to $16.7 \%, 15.1 \%$ and

Table 1. The issues distribution of public appeals.

\begin{tabular}{cccccccc}
\hline & $\begin{array}{c}\text { Demolition and } \\
\text { requisition }\end{array}$ & $\begin{array}{c}\text { Municipal } \\
\text { construction }\end{array}$ & $\begin{array}{c}\text { Environment } \\
\text { protection }\end{array}$ & $\begin{array}{c}\text { Medical } \\
\text { hygiene }\end{array}$ & Education & $\begin{array}{c}\text { Social } \\
\text { security }\end{array}$ & $\begin{array}{c}\text { Rural } \\
\text { agriculture }\end{array}$ \\
\hline Post/piece & 32,235 & 50,706 & 35,473 & 24,678 & 44,277 & 31,380 & 69,277 \\
Percentage/\% & 1.13 & 23.81 & 16.66 & 11.59 & 20.79 & 14.73 & 32.53 \\
Post/piece & Traffic & Employment & $\begin{array}{c}\text { Enterprise } \\
\text { affairs }\end{array}$ & $\begin{array}{c}\text { Culture and } \\
\text { entertainment }\end{array}$ & Corruption & Other issues \\
Percentage/\% & 44,291 & 68,303 & 58,022 & 14,466 & 62,852 & 11,757 \\
\hline
\end{tabular}

PS: $\mathrm{N}=212,985$. 
$14.7 \%$ respectively. While the number of posts of attention to health care, culture and entertainment, and other issues is less than 25,000 respectively.

The trend of public appeal issues is also worthy of attention. Table 2 shows the distribution and trends of citizen claims in the three categories of issues from 2008 to 2013 through radar charts. It can be found that the postings of almost all issues have increased substantially. The five major issues of employment, rural agriculture, corruption, urban construction and transportation are not only the top posts in all issues, but also the fastest growth in postings. Though the attention to the four major issues of corporate affairs, education, social security and land acquisition and demolition have a slight decline in 2011, they have shown a growth trend in the rest of the years. The attention to environmental protection issues has declined in 2011 and 2012, but it has increased significantly to the 2010 level in 2013. While the attention to cultural entertainment and other issues have declined.

Interpret Table 1 in terms of time. First, as shown in Table 2, from the aspect of the posting month selection, the first half year of the post is higher than the second half. The number of posts was the highest in March, and was continued its high trend from April to July. The number of posts from August to October and January was lower, but it was increased a little from November to December. Second, from the aspect of public percentage of claims in working days, the percentage is highest on Thursday, the lowest on weekends, and keeps a stable high level from Monday to Wednesday. Third, from the perspective of posting time, the 10 a.m., 4 p.m., and 9 p.m. are the three peak times in posting. Specifically, the ratio of claims is highest from 9 to 11 a.m., then from 2 p.m. to 4 p.m., and from 8 p.m. to 10 p.m. These findings are closely related to China's political cycle and institutional arrangements for government responses. Judging

Table 2. Annual distribution of the number of public claims on the online political platform.

\begin{tabular}{cccccc}
\hline & $\begin{array}{c}\text { Secretary } \\
\text { (piece) }\end{array}$ & $\begin{array}{c}\text { Percentage } \\
(\%)\end{array}$ & $\begin{array}{c}\text { Governor/Mayor/ } \\
\text { Chairman (piece) }\end{array}$ & $\begin{array}{c}\text { Percentage } \\
(\%)\end{array}$ & Sum up \\
\hline 2006 & 98 & 66.2 & 50 & 33.8 & 148 \\
2007 & 427 & 69.7 & 186 & 30.3 & 613 \\
2008 & 11,222 & 71.7 & 4438 & 28.3 & 15,660 \\
2009 & 18,584 & 71.6 & 7355 & 32.2 & 31,175 \\
2010 & 21,132 & 67.8 & 10,043 & 37.5 & 30,018 \\
2011 & 18,754 & 62.5 & 11,264 & 35.3 & 32,919 \\
2012 & 21,311 & 64.7 & 11,608 & 36.8 & 43,192 \\
2013 & 27,317 & 63.2 & 15,875 & & 31.7 \\
First half & 22,773 & 68.3 & 10,548 & 33.5 & 212,985 \\
year of 2014 & & 66.5 & 71,367 & 321
\end{tabular}

http://leaders.people.com.cn/GB/178291/218130/370358/index.html. 
from the choice of the month, the citizen expression opportunities and government response responsibilities created by the "two sessions" stimulated the expression of citizen appeals in cyberspace, and the year-end assessment of party and government organizations also provided an opportunity for the expression of citizen appeals. Judging from the choice of working days and hours, citizens mainly express their demands during government working hours.

Interpret Figure 2 [5] in terms of space. First, from the total posting scale, Tianjin, Henan, and Anhui provinces have more than 15,000 posts respectively, ranking the top three in the country; the number of posts in Shandong, Shanxi and other three provinces have varied from 10,000 to 15,000. The number of posts in Hebei, Hubei and other three provinces is moderate, about 5000 10,000; the number of posts in Hainan, Ningxia, Qinghai, Tibet, Chongqing, Zhejiang and Yunnan provinces is less than 3000 each, ranking the country's last seven. Throughout the country, netizens in the central region have the largest scale of expression, and netizens in North China and Northwest China are also very active. While relatively speaking, netizens in the southeastern coastal provinces and ethnic minority areas have fewer claims. And the total amount of postings reflects the regional differences in the scale of citizens' claims. In fact, the issues of concern to cyberspace in different regions are very different. In environmental protection issues, Beijing, Tianjin, Shanghai, Ningxia and Xinjiang have the highest proportion of attention. And the proportion in most northern provinces and Guangdong, Guangxi and Hainan are also high, which is fully affected by the deterioration of environmental conditions (especially air pollution) and citizens' increasing requirements for environmental quality. The provinces with the highest proportion of concerns about corruption are Liaoning, Tianjin, Henan, Anhui and Zhejiang, and Shandong, Shandong, Shaanxi, Jiangsu, Fujian, Sichuan and Guizhou are second echelon. Coastal provinces and some relatively developed provinces in the central and western regions have a higher proportion
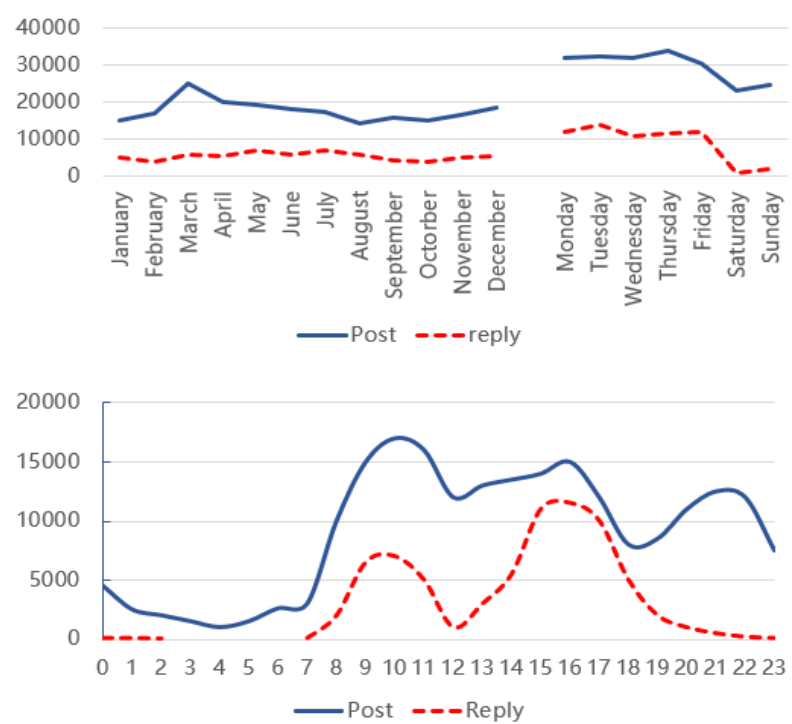

Figure 2. Time distribution of public appeals and government responses. 
of concerns about corruption, which not only reflects the complex relationship between economic development and corruption, but also reflects the decline of citizens' tolerance for corruption in economically developed regions. The provinces most concerned about the issue are ethnic minority provinces, such as Inner Mongolia, Xinjiang, and Tibet. While netizens with more surplus labor in the northeastern, northern, northwestern, and southwestern regions have also expressed higher employment-related claims.

This article also performs a text sentiment analysis of all texts. Text sentiment analysis is a process of analyzing, processing, summarizing and reasoning subjective texts with emotion. It generally includes the extraction of emotional information, the classification of emotional information, and the retrieval and induction of emotional information. The sentiment analysis of political texts can effectively learn the political and emotional tendencies of political actors, which influence the specific mode of political interaction. This paper uses text sentiment analysis to analyze the political sentiment of all netizens posting, and summarizes the netizens' postings into five categories: "strong negative, weak negative, neutral, weak positive, strong positive", which reflect the emotional inclination and intensity of political discourse expression in netizens' posting.

Most citizens take a positive emotional expression in their claims. The proportion of strong positive and weak positive expressions to the government is $7.6 \%$ and $47.8 \%$. While $35.4 \%$ of citizens' demands are neutral. And the proportion of negative postings is $9.2 \%$, of which only $0.6 \%$ take a strong negative emotional expression. The authoritarian political culture of China has a lasting impact on the expression of citizen appeal (Shi, 2001). The authoritarian political culture shapes citizen's reliance and awe on political authority, and the collective use of positive emotions and authority interaction is its inevitable consequence. Figure 3 [4] also shows the emotional orientation of citizens when expressing different issues. The emotional orientation of cultural entertainment, municipal construction and education are the most active. The emotional tendency of demolition, environmental protection and employment are also very positive. The emotional sentiment of traffic, rural agriculture, enterprises, corruption and other issues is neutral. While citizens' emotions in expressing their appeals on health care and social security issues are the most negative [4].

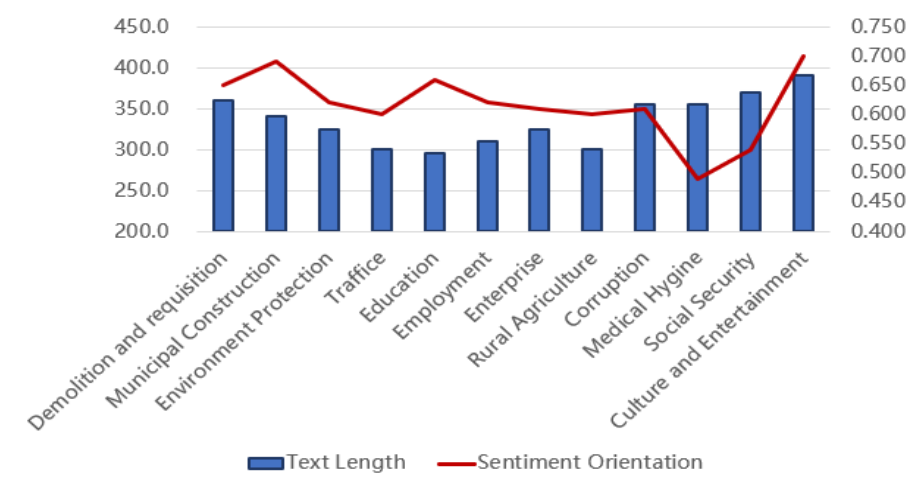

Figure 3. The length of text and sentiment of public appeal. 
Finally, based on the above data, this paper uses Logit regression to examine the impact of citizens' issues, political appeals and time and space on the government's response to citizens' demands on the basis of statistical control. The dependent variable of the model is government responsiveness, that is, the government responds or does not respond to citizens' demands, taking a 0 - 1 measurement. As shown in Table 3 [5], the model (1) is the benchmark model, which only includes the provincial dummy variables; the model (2) incorporates

Table 3. Government responsive interpretation model: Based on Logit regression.

\begin{tabular}{|c|c|c|c|c|c|c|c|}
\hline \multirow[b]{2}{*}{$\begin{array}{l}\text { Independent } \\
\text { variable }\end{array}$} & \multirow{2}{*}{$\begin{array}{c}\text { Model(1) } \\
\text { Regression } \\
\text { coefficients } \\
\text { (standard error) }\end{array}$} & \multicolumn{2}{|c|}{ Model(2) } & \multicolumn{2}{|l|}{ Model(3) } & \multicolumn{2}{|l|}{ Model(4) } \\
\hline & & $\begin{array}{c}\text { Regression } \\
\text { coefficients } \\
\text { (standard error) }\end{array}$ & $\begin{array}{l}\text { Happening } \\
\quad \text { ratio }\end{array}$ & $\begin{array}{c}\text { Regression } \\
\text { coefficients } \\
\text { (standard error) }\end{array}$ & $\begin{array}{l}\text { Happening } \\
\quad \text { ratio }\end{array}$ & $\begin{array}{c}\text { Regression } \\
\text { coefficients } \\
\text { (standard error) }\end{array}$ & $\begin{array}{l}\text { Happening } \\
\quad \text { ratio }\end{array}$ \\
\hline \multicolumn{8}{|c|}{ Reference(2008) } \\
\hline 2009 & & $1.14(0.06)^{\star \star \star}$ & 3.14 & $1.11(0.06)^{\star * \star}$ & 3.03 & $1.07(0.06)^{\star \star \star}$ & 2.91 \\
\hline 2010 & & $1.48(0.05)^{* * *}$ & 4.39 & $1.50(0.06)^{\star * *}$ & 4.48 & $1.36(0.06)^{* * *}$ & 3.90 \\
\hline 2011 & & $3.08(0.05)^{\star * *}$ & 21.66 & $3.08(0.05)^{\star \star \star}$ & 21.67 & $2.93(0.05)^{* * *}$ & 18.65 \\
\hline 2012 & & $3.49(0.05)^{\star * *}$ & 32.83 & $3.48(0.05)^{* * *}$ & 32.55 & $3.32(0.05)^{* * *}$ & 27.54 \\
\hline 2013 & & $3.36(0.05)^{\star * *}$ & 28.67 & $3.38(0.05)^{\star \star \star}$ & 29.30 & $3.23(0.05)^{\star * \star}$ & 25.20 \\
\hline 2014 & & $3.17(0.05)^{\star * \star}$ & 23.76 & $3.20(0.05)^{* * *}$ & 24.64 & $3.06(0.05)^{* * *}$ & 21.42 \\
\hline \multicolumn{8}{|l|}{ Issues (others) } \\
\hline Demolition and acquisition & & & & $-0.07(0.02)^{* * *}$ & 0.93 & $-0.14(0.02)^{* * \star}$ & \\
\hline Municipal construction & & & & $0.61(0.02)^{\star * \star}$ & 1.84 & $0.52(0.02)^{\star \star \star}$ & \\
\hline Environment protection & & & & $-2.60(0.02)^{\star * \star}$ & 0.07 & $-2.66(0.03)^{\star * *}$ & \\
\hline Educ+I45:O54ation & & & & $0.08(0.02)^{* * *}$ & 1.08 & $-0.02(0.02)$ & \\
\hline Employment & & & & $-0.28(0.02)^{\star * *}$ & 0.75 & $-0.38(0.02)^{* \star *}$ & \\
\hline Enterprise affairs & & & & $0.13(0.02)^{\star * *}$ & 1.14 & $0.06(0.02)^{* *}$ & \\
\hline Rural agriculture & & & & $0.15(0.02)^{\star * \star}$ & 1.16 & $0.02(0.001)$ & \\
\hline Corruption & & & & $0.20(0.02)^{* * *}$ & 1.22 & $0.08(0.02)^{\star * *}$ & \\
\hline Medical hygiene & & & & $0.32(0.02)^{\star * *}$ & 1.38 & $0.22(0.02)^{\star * *}$ & \\
\hline Social security & & & & $0.20(0.02)^{\star * \star}$ & 1.22 & $0.10(0.02)^{\star \star \star}$ & \\
\hline Culture and entertainment & & & & $0.12(0.02)^{\star * \star}$ & 1.13 & $0.01(0.03)$ & \\
\hline Secretary (Governor) & & & & & & $0.09(0.01)^{\star \star \star *}$ & \\
\hline Posting in real-name & & & & & & $0.18(0.01)^{\star \star \star}$ & \\
\hline Sentimental tendency & & & & & & $-0.05(0.01)^{* * \star}$ & \\
\hline Ln (tsxt length) & & & & & & $0.32(0.01)^{\star * *}$ & \\
\hline Constant term & $-8.58(1.00)^{\star * *}$ & $-11.47(1.00)^{* * *}$ & 0.00 & $-11.38(1.00)^{* * *}$ & 0.00 & $-12.68(1.00)^{* * *}$ & 0 \\
\hline Province dummy variable & yes & yes & & yes & & yes & \\
\hline Log likelihood & -90884.1 & -79245.7 & & -69900.8 & & -69257.7 & \\
\hline Pseudo R2 & 0.3239 & 0.4105 & & 0.4703 & & 0.4752 & \\
\hline $\mathrm{N}$ & 212224 & 212224 & & 208272 & & 208272 & \\
\hline
\end{tabular}

PS: 1) References for categorical independent variables in the table are presented in parentheses; 2$)^{* * *}$ represents a significant level of 0.001 , ${ }^{* *}$ represents a significant level of 0.01 , and ${ }^{*}$ represents a significant level of 0.05 
the time factor to examine the government's responsiveness cross-year trends; the model (3) adds the issue-attention of citizen appeals to examine choices of government responsive issues. Model (4) incorporates the expression of citizen appeal. See Table 3 for the results of Logit regression. Overall, the model has a high degree of fitting, and the model (1) to model (4) can be used to explain the government responsiveness from $32.4 \%$ to $47.5 \%$.

\subsubsection{Time and Space Differences in Government Responsiveness}

First, the spatial differences and cross-time changes in government responsiveness should be examined. Model (1) and model (2) respectively examined the impact of province and year factors on government responsiveness. For the sake of comparison, Table 4 [6] shows the response rate of the party and government agencies in the provinces to the citizens' claims, that is, the proportion of government responses to the total number of posts. The deeper the color, the higher the government response rate. In terms of response rate, Anhui, Henan, Sichuan and Tibet have a response rate of more than 50\%, ranking first in the country; Tianjin, Shanxi, Hubei, Guizhou and Yunnan have a government response rate of $25 \%$ to $50 \%$, basically reaching the national average. The response rate of most other provinces remained at $10 \%-25 \%$; the response rates of the eight provinces of Heilongjiang, Hebei, Jiangsu and Guangdong are very low. In summary, the low level of network government responsiveness at this stage is mainly due to the lack of government arrangements for responding to citizens' demands in some provinces. Model (2) examines the trends of government responsiveness in different years after controlling for provincial differences. Compared with 2008, the government responsiveness has continued to increase significantly from 2009-2012. The government's response to citizen claims in 2009 was as 3.14 times high as in 2008, and the government's response to citizen

Table 4. Annual distribution of the number of public claims on the online political platform.

\begin{tabular}{cccccc}
\hline & $\begin{array}{c}\text { Secretary } \\
\text { (piece) }\end{array}$ & $\begin{array}{c}\text { Percentage } \\
(\%)\end{array}$ & $\begin{array}{c}\text { Governor/Mayor/Chairman } \\
\text { (piece) }\end{array}$ & $\begin{array}{c}\text { Percentage } \\
(\%)\end{array}$ & $\begin{array}{c}\text { Sum } \\
\text { up }\end{array}$ \\
\hline 2006 & 98 & 66.2 & 50 & 33.8 & 148 \\
2007 & 427 & 69.7 & 186 & 30.3 & 613 \\
2008 & 11,222 & 71.7 & 4438 & 28.3 & 15,660 \\
2009 & 18,584 & 71.6 & 7355 & 28.4 & 25,939 \\
2010 & 21,132 & 67.8 & 10,043 & 32.2 & 31,175 \\
2011 & 18,754 & 62.5 & 11,264 & 37.5 & 30,018 \\
2012 & 21,311 & 64.7 & 11,608 & 35.3 & 32,919 \\
2013 & 27,317 & 63.2 & 15,875 & 36.8 & 43,192 \\
First half & 22,773 & 68.3 & & & 31.7 \\
year of 2014 & & 66.5 & 10,548 & 33,321 \\
Sum up & 141,618 & & & 31,367 & 212,985 \\
\hline
\end{tabular}


claims in 2012 was as 32.82 times high as in 2008. Relatively speaking, the government's response to citizen appeals in 2013 and 2014 was basically the same as in 2012, which is about 20 times that of 2008. After more than four years of rapid development, the government responsiveness in cyberspace has gradually entered a stable operation phase in 2012. Although the model (4) shows that the cross-time growth effect of the government responsive has slightly reduced after controlling the issue attention and expression, it still has a great impact, as the government responsiveness in 2012-2014 is more than 20 times that of 2008.

\subsubsection{Topic Selection of Government Responsiveness}

The difference of regions and years in government responsiveness reflects the systematic differences in government responsiveness system construction. The difference in response rates between provinces stems from their differences in the degree of emphasis on online public opinion and the construction of response mechanisms, while the annual differences reflect the impact of the government's response to institution improvements in online public opinion. In addition, the government's responsiveness to citizen appeals on different topics is also worthy of attention. This actually reflects the essential characteristics of the government's responsiveness, that is, what appeals and whose appeals does the government respond to. As analyzed above, different issues appeal represents the different social groups' interests and opinions, and the difference of government's responsiveness to different issues reflects its willingness and behavior to respond to the interests of different social groups.

Model (3) identifies the government's selective response to different issues. Compared with the government's responsiveness to other issues, the three issues of environmental protection, employment and land acquisition and demolition responsive is significantly low, with only $7 \%, 75 \%$ and $93 \%$ of other issues responsive respectively. Relatively speaking, the government responsiveness to nine topics, such as urban construction and health care, is significantly higher than that of other issues. Among them, urban construction, transportation, enterprises, culture and entertainment and other economic issues are generally more responsive. And the responsiveness of health and welfare, social security, education and other issues concerned about people's livelihood and welfare is mutual, and the responsiveness of corruption and agricultural and rural issues is relatively high. However, after the model (4) controls the factors of expressions of appeal, the government's responsiveness to the three issues of environmental protection, employment and land acquisition and demolition has become even lower, while the government's responsiveness to education, agriculture, rural and cultural entertainment issues is no longer statistically significant compared with other issues. The responsiveness of the other six issues, such as urban construction and health care, is still significantly higher than that of other issues, but the impact has declined.

\subsubsection{Expressions of Citizens Appeal and Government Responsiveness}

The model (4) shows that the length of the text, the emotional orientation, post- 
ing in real or fake name, posting to the secretary or the governor of the citizen appeal significantly influence the government responsiveness. However, compared with the influence of time and space factors and the attribution of issues, the influence of expressions of citizens appeal is very limited. Specifically, the secretary is more responsive to citizens' demands and is as 1.1 times as the governor's response. Real-name postings in receive $20 \%$ higher government response than anonymous postings. The more positive a citizen's appeal is, the lower the government response is. Every one point increased in the positive sentiment of citizen appeals, every $0.05 \%$ reduced in the government's responsiveness. Consistent with expectations, the longer the text of citizen appeals, the higher the possibility that the government will respond to its claims.

\subsection{Research on Big Data Management of Grass-Roots Government}

The grass-roots government often holds a large amount of data about people's livelihood. By using these big data, it can significantly improve people's livelihood and strengthen the construction of a service-oriented government. According to the big data-based government governance decision-making model, the big data government governance application framework can be launched. That is, taking various data sources as the source, based on data collection, using data analysis as a means, and taking ability promotion as the point of strength. Therefore, as Figure 4 showed, to obtain the goal of modernization of social management and public service capabilities and utilization of social autonomy modernization, and to achieve the purpose of comprehensively improving the modernization level of government governance capabilities.
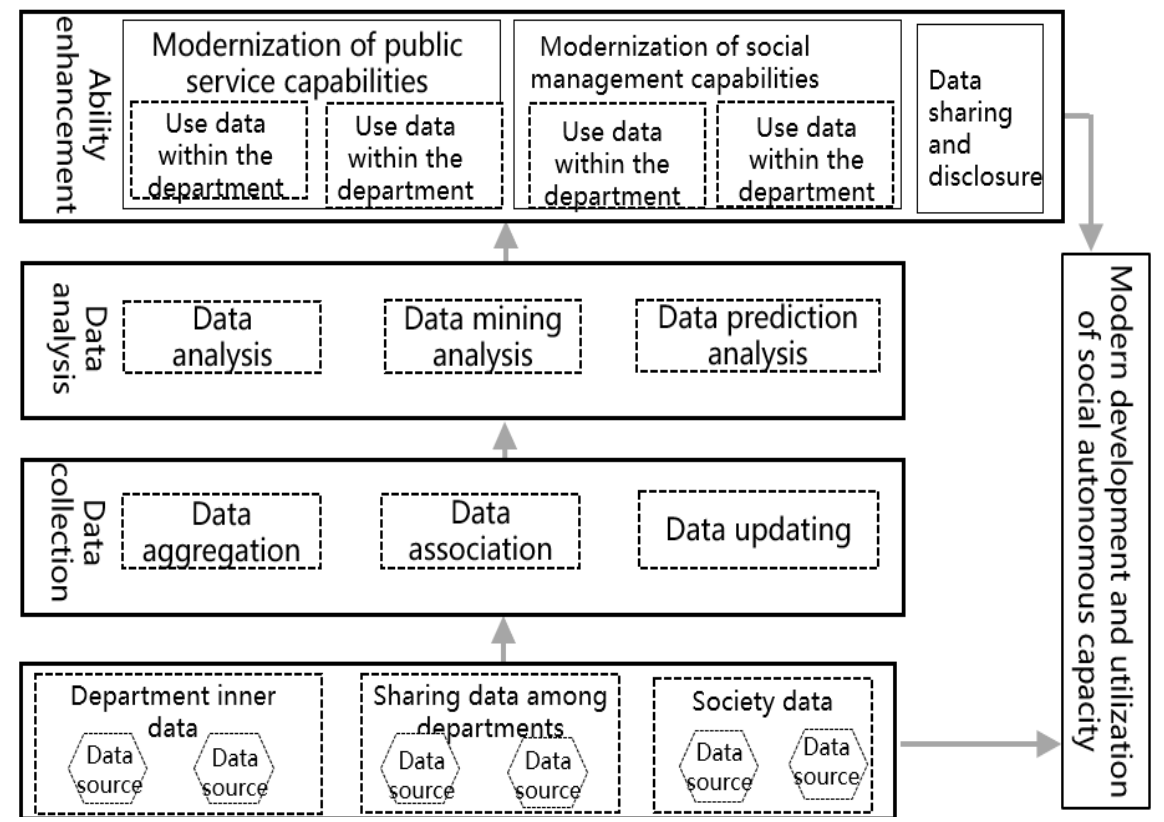

Figure 4. The application framework of big data promoting the modernization of government capacity. 
Management in Beijing Dongcheng District is an example of application framework of big data government governance. Since 2006, Dongcheng District has applied the data analysis capabilities of the Big Data Framework to rapidly improve community public health service capabilities. It uses data sharing and publicity to promote the favorable mutual interaction between community residents and community health service institutions and general practitioners, to achieve self-scientific management of the health status of community residents. Through the correlation analysis of the chronic disease impact factors of residents in the district, it provides regional disease early warning services, and promotes the innovation of grassroots public service models. The big data carried by this system, covering about 1 million personal medical files of 38 community health service centers and more than 2.2 million electronic medical records, updated at a rate of more than 3000 records per day.

The management of Dongcheng District applies he big data analysis framework and uses the big data collected by the district community health service system to realize the scientific judgment of the chronic disease impact factors of community residents in the Internet age. The data source of the big data of the community health service system is a medical file that records the daily habits of various chronic diseases patients, and provides a data basis for analyzing the correlation of the chronic disease impact factors of community residents. Based on years of medical history files and electronic medical records, the relevant departments of Dongcheng District organized the construction of decision trees and other analytical models, to analyze the correlation between hypertension and coronary heart disease and several daily habits. Through analysis, the variables that have important influences are: age, education level, dietary taste, weight, age segment related with drinking, whether drinking or not and the level of alcohol. The results of the model analysis [7] showed the relationship between residents' daily habits and high blood pressure and coronary heart disease they had, which provides guidance for community doctor services.

\section{Research Conclusions}

In summary, the application of big data in Chinese government governance is very effective. The government governance model based on big data will definitely make the Chinese government become an intelligent and service-oriented government. However, the application of big data has encountered obstacles, which mainly lie in insufficient sharing, incomplete services, and shortage of law to rely on. Therefore, to deal with these three obstacles, recommendations are as follows.

\subsection{Sharing and Opening: Unblock "Internet + Government Services" Data Stream}

Sharing and opening is an inevitable requirement of modern government. The traditional closed mechanism of government agencies brings the "fragmentation of administrative organization structure, data resources and public service 
supply". The first is information sharing of cross-regional, cross-level and cross-regions. The second is the open data. The big data of online government contains great value, which provides more possibilities for speeding up and increasing efficiency of "Internet + government services", and also provides guidance for the value-added utilization of government information resources.

\subsection{Optimize Services: Improve the Supply Capacity of "Internet + Government Services"}

The contradiction between people's growing demands for online government services and the unbalanced and uneven development is the basic contradiction currently faced by "Internet + government services". First is to expand the coverage of online services. Thus, to ensure the government services meet all the needs and are online all the way. The second is to gradually expand the depth of online services. From the practical point of view, the depth of online services can be divided into four stages of development: information disclosure, form downloading, online booking and online processing. The differences in the development of different regions should be clarified in the design of the system.

\subsection{Transparency and Rule of Law: Optimizing the Environment of the Development of "Internet + Government Services"}

The Internet + government service's promotion process can only protect the construction of law government by following the basic concepts of transparency and rule of law. First, actively carry out the application of electronic licenses, electronic official documents, electronic signatures and other applications in "Internet + Government Services". Clearly define their legal status in "Internet + Government Services" as soon as possible. Second, carry out regulatory legislation on data flow and utilization as soon as possible. Increase protection of state secrets, trade secrets, personal privacy and intellectual property rights.

\section{Conflicts of Interest}

The authors declare no conflicts of interest regarding the publication of this paper.

\section{References}

[1] Joseph, R.C. and Johnson, N.A. (2013) Big Data and Transformational Government. IT Professional Magazine, 15, 53. https://doi.org/10.1109/MITP.2013.61

[2] Bertot, J.C., Gorham, U., Jaeger, P.T., Sarina, L.C. and Choi, H. (2014) Big Data, Open Government and E-Government: Issues, Policies and Recommendations. Information Polity, No. 19. https://doi.org/10.3233/IP-140328

[3] Zhao, W., Qin, B. and Liu, T. (2010) Text Emotion Analysis. Journal of Software, No. 8.

[4] Meng, T.G. and Li, F. (2015) Political Interaction in Cyberspace: Citizen Appeals and Government Responsiveness-Big Data Analysis Based on Global Network Policy Platform. 
[5] http://leaders.people.com.cn/GB/178291/218130/370358/index.html

[6] Chen, P. (2006) A New Model of Gridded Urban Management.

[7] Zhang, Y.J. (2012) Grid Space and Government Management Innovation. 